\title{
GPU-Monte Carlo based fast IMRT plan optimization
}

\author{
Yongbao Li, Zhen Tian, Feng Shi, Steve Jiang, Xun Jia \\ Department of Radiation Oncology, UT Southwestern Medical Center, Dallas, TX, USA.
}

Received March 19, 2014; Published Online April 08, 2014

[Presented at the Young Investigator's Symposium at the 2014 Annual Meeting of

Southwest Chapter of American Association of Physicists in Medicine (AAPM) in San Antonio, Texas, USA]

\section{Conference Proceeding}

\begin{abstract}
Purpose: Intensity-modulated radiation treatment (IMRT) plan optimization needs pre-calculated beamlet dose distribution. Pencil-beam or superposition/convolution type algorithms are typically used because of high computation speed. However, inaccurate beamlet dose distributions, particularly in cases with high levels of inhomogeneity, may mislead optimization, hindering the resulting plan quality. It is desire to use Monte Carlo (MC) methods for beamlet dose calculations. Yet, the long computational time from repeated dose calculations for a number of beamlets prevents this application. It is our objective to integrate a GPU-based MC dose engine in lung IMRT optimization using a novel two-steps workflow.
\end{abstract}

Methods: A GPU-based MC code gDPM is used. Each particle is tagged with an index of a beamlet where the source particle is from. Deposit dose are stored separately for beamlets based on the index. Due to limited GPU memory size, a pyramid space is allocated for each beamlet, and dose outside the space is neglected. A two-steps optimization workflow is proposed for fast MC-based optimization. At first step, a rough dose calculation is conducted with only a few number of particle per beamlet. Plan optimization is followed to get an approximated fluence map. In the second step, more accurate beamlet doses are calculated, where sampled number of particles for a beamlet is proportional to the intensity determined previously. A second-round optimization is conducted, yielding the final result.

Results: For a lung case with 5317 beamlets, 105 particles per

Presenting author: Yongbao Li; Department of Radiation Oncology, UT Southwestern Medical Center, Dallas, TX, USA.

Cite this article as:

Li Y, Tian Z, Shi F, Jiang S, Jia X. GPU-Monte Carlo based fast IMRT plan optimization. Int J Cancer Ther Oncol 2014;

2(2):020244. DOI: 10.14319/ijcto.0202.44 beamlet in the first round, and 108 particles per beam in the second round are enough to get a good plan quality. The total simulation time is $96.4 \mathrm{sec}$.

Conclusion: A fast GPU-based MC dose calculation method along with a novel two-step optimization workflow are developed. The high efficiency allows the use of MC for IMRT optimizations.

Innovation/Impact: It has long been desired to use Monte Carlo (MC) simulations for beamlet dose calculations in IMRT plan optimization, due to the well accepted dose calculation accuracy, particularly in cases with high levels of inhomogeneity, e.g. lung. Yet, long computation time and large memory requirement prevent this. In this work, we developed a GPU-based beamlet dose calculation method using MC simulations, as well as a novel two-steps optimization workflow, for fast IMRT plan optimization.

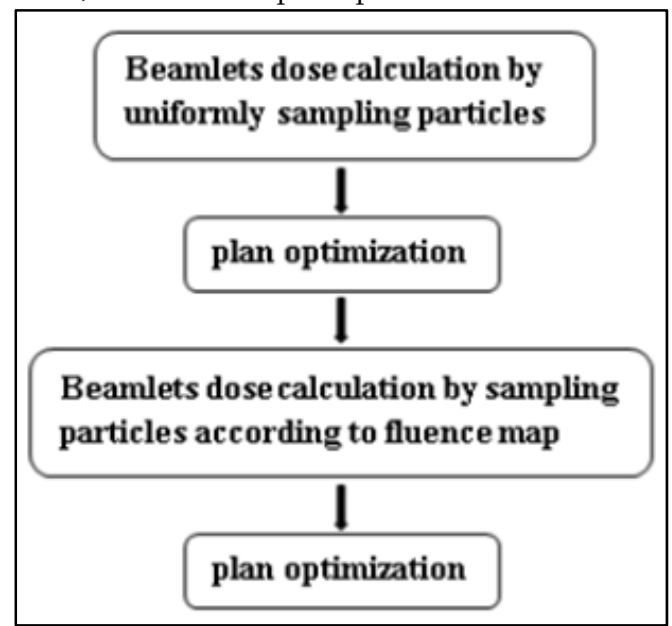

FIG. 1: The two-step optimization workflow. 


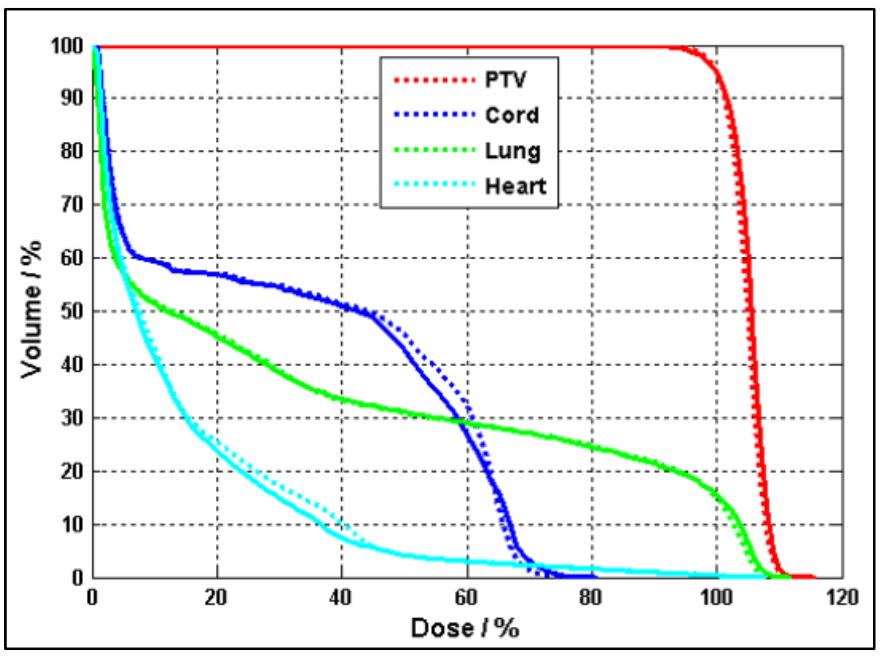

FIG. 2: Comparison of DVHs for a lung case. Solid, optimized plan in Eclipse. Dash, the same plan computed using a MC method.

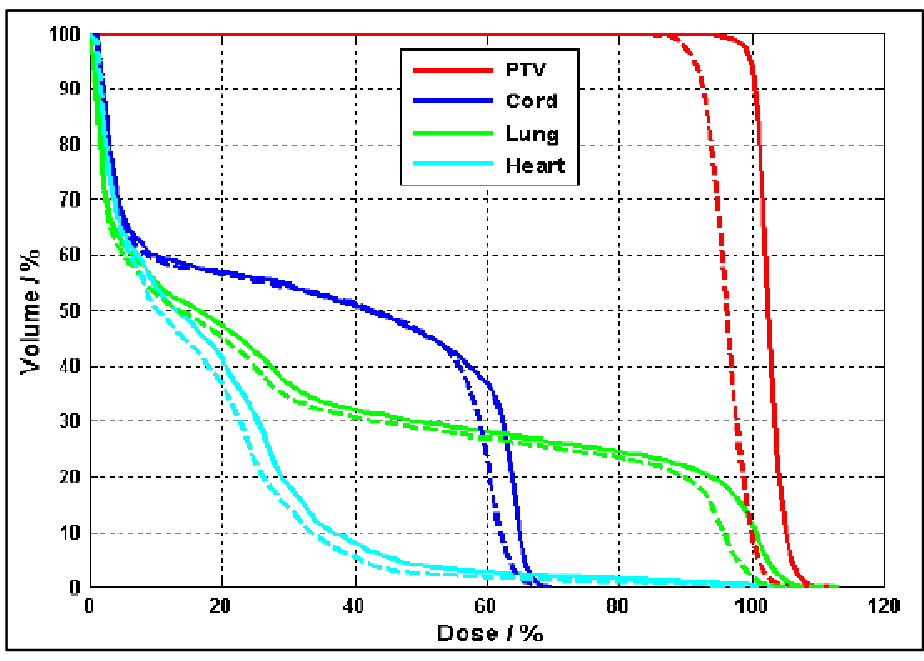

FIG. 3: DVHs optimized using MC methods.

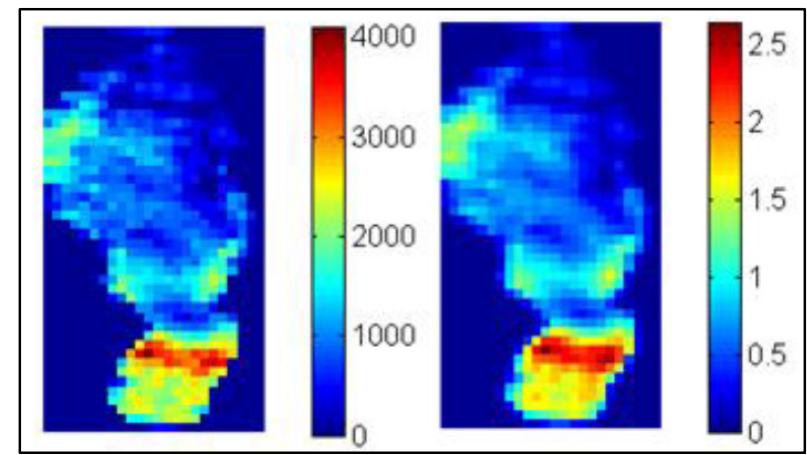

FIG 4: (left) approximated fluence map in the first step. (right) sampled number of particles per beamlet (in unit $10^{5}$ ). 